\title{
Empleabilidad y formación de inmigrantes ${ }^{1}$
}

\author{
Félix Etxeberria, Elisabet Arrieta, \\ José Garmendia y José Ignacio Imaz \\ Universidad del País Vasco
}

\section{Resumen}

El siguiente texto resume una investigación que se ha realizado durante los cursos 2006/7 y 2007/8 para analizar la empleabilidad de los inmigrantes en la Unión Europea. Para ello, se han llevado a cabo estudios en España, Francia y Países Bajos. El primer objetivo de la investigación ha sido descubrir las claves que permiten explicar mejor las circunstancias que favorecen un mayor acceso al empleo de los inmigrantes y una mayor continuidad en el mismo. El segundo objetivo, ha consistido en elaborar una serie de recomendaciones para fomentar la empleabilidad de los inmigrantes y una mayor y mejor integración en la sociedad europea. Nuestro artículo anticipa los resultados del equipo de investigación español.

Palabras ClaVe: inmigración, inserción laboral, empleabilidad, formación, Unión Europea, España, País Vasco.

\section{Summary}

This text brings together the results of research undertaken during 2006/7 and 2007/8 in order to analyse the employability of immigrants in the European Union. To this end, studies were carried out in Spain, France and the Netherlands. The first goal of the research was to discover a better description of the circumstances favouring access to the labour market for immigrants and their continuity in employment. The second aim was to draw up a series of recommendations to boost employability amongst immigrants as well as greater and better integration into European society. Our article here deals with the results of the Spanish research team.

KEY WORDS: immigration, entering the labour market, employability, vocational training, the European Union, Spain, the Basque Country.

\section{Introducción}

La Unión Europea busca en estos últimos años una política común en materia de inmigración, siendo uno de los objetivos de esta política la integración de los inmigrantes. En este sentido, es evidente que una de las claves más importantes para la integración pasa por el empleo. El siguiente texto es parte de una investigación más amplia que investiga el tema de "la empleabilidad de los inmigrantes en la Unión Europea”. 
Se ha analizado la situación respecto al empleo de los inmigrantes, con el objetivo de definir indicadores de empleabilidad (factores que pueden favorecer el acceso a un empleo y su posterior mantenimiento), como parte del proceso de formación que puede facilitar en un futuro la integración de las personas inmigrantes.

Nuestro estudio se ha realizado en Donostia-San Sebastián, ciudad que cuenta en el 2008 con un porcentaje de población extranjera del $5 \%$, muy por debajo todavía de otras ciudades españolas que superan la tasa del 10\% e incluso del 15\%. De todas formas, habría que señalar también que la llegada de inmigrantes a partir del año 2000 está siendo constante e importante, por lo las perspectivas a medio plazo apuntan hacia un crecimiento continuado de la inmigración, que podría situarse en torno al $7 \%$ de la población en el año 2010 (en una población de 200.000 habitantes en la ciudad, y de 400.000 en la comarca o en el "área metropolitana").

Para la ciudad de San Sebastián, el primer nivel que condiciona su política en materia de inmigración ${ }^{2}$ es la Comunidad Autónoma del País Vasco (CAPV). El I Plan Vasco de Inmigración fue aprobado en el 2003 y ha sido el texto que ha regido la política de inmigración en la CAPV durante el período 2003-2005. El Plan ha impulsado la creación de diversas estructuras y recursos, como el Observatorio Vasco de Inmigración (Ikuspegi ${ }^{3}$ ), el Foro para la integración y participación social de los ciudadanos y ciudadanas inmigrantes, el programa de atención socio-jurídica a personas inmigrantes (Heldu), la Red de Acogida de Base Municipal y el Centro Coordinador de Iniciativas de Educación y Mediación Intercultural (Biltzen). El actual plan, el II Plan Vasco de Inmigración (2007-2009), promueve la inserción de los trabajadores no nacionales en el mercado laboral para conseguir su "plena integración en la sociedad".
Los críticos con estos dos planes del Gobierno Vasco resaltan el hecho de que presentan grandes intenciones (se habla de ciudadanía inclusiva, ciudadanía europea y otros aspectos interesantes), pero que luego hay un salto entre esas intenciones y la capacidad de concretarlas, de acercarlas a la realidad de todos los días.

El siguiente nivel marco es el del Estado Español, y en este caso nos parece también importante referirnos al marco legal que se impone para los inmigrantes. Actualmente, la Ley de Extranjería (R.D. 2393/2004) define básicamente tres formas de acceso a la regularidad, a los permisos de trabajo y residencia: el régimen general, el arraigo laboral y el arraigo social.

1.- El régimen general: el elemento novedoso que aporta el nuevo Reglamento se sitúa en el inicio del trámite, en la fase en la que el empresario tiene que acudir al Instituto Nacional de Empleo, INEM, para saber si el puesto que le ofrece al trabajador extranjero está "libre", es decir, si no hay trabajadores de nacionalidad española en paro que puedan ocuparlo.

2.- El arraigo laboral: es un sistema nuevo. Para poder regularizarse por ese sistema se requiere demostrar que se lleva en el país como mínimo dos años en situación irregular y que se ha trabajado como mínimo un año, habitualmente en la economía sumergida. Con este sistema se pretende combatir la inmigración clandestina, pero para poder acceder a él hay que demostrar que se llevan dos años como mínimo en situación clandestina.

3.- El arraigo social: es un sistema parcialmente nuevo. Se requiere demostrar una estancia previa de tres años, tener un contrato de trabajo de un año de duración y tener familiares de primer grado con permiso de residencia en vigor o, en su defecto, disponer 
de un informe hecho por el ayuntamiento, sobre el grado de inserción social en la ciudad.

Evidentemente, estas tres vías no favorecen un tránsito rápido y sencillo de la irregularidad a la regularidad. Más bien constituyen un sistema diseñado como una carrera de obstáculos que dura 7-8 años, similar a un sistema de existencia provisional prolongada que tiene, a su vez, una incidencia directa en materia de vivienda. Paradójicamente, en esta carrera de obstáculos, es la propia Administración la que organiza esos itinerarios que combinan lo regular y lo irregular. ${ }^{4}$

En este contexto demográfico y jurídico, los objetivos de nuestra investigación han sido: (1) descubrir las claves que permiten explicar mejor las circunstancias que favorecen un mayor acceso al empleo de los inmigrantes y una mayor continuidad en el mismo, y (2) elaborar una serie de recomendaciones para fomentar la empleabilidad de los inmigrantes y una mayor integración en la sociedad vasca.

\section{2- Metodología}

Una de las mayores dificultades a la que se enfrenta un estudio europeo de este tipo es la de coordinar diferentes formas de entender la investigación. Por eso, las primeras reuniones celebradas en el verano del 2006 se dedicaron a coordinar los objetivos, la metodología y el cronograma.

Se decidió estudiar el tema a nivel de ciudad-área metropolitana, por lo que en nuestro caso eso suponía acotar el marco geográfico de la investigación a San Sebastián. El siguiente paso fue construir una batería de posibles indicadores que condicionan la empleabilidad de los inmigrantes. Una vez construida la batería, nos reunimos en otoño del 2006 con agentes sociales que trabajan con el tema para validar la misma (técnicos municipales, trabajadores de ONG, técnicos del INEM, Colegio Oficial de Educadores Sociales...) (Tabla 1).

Con el listado de posibles indicadores más importantes en la mano, elaboramos un cuestionario en el que se debería responder el grado de importancia que tuviera cada indicador para el entrevistado (desde "nada importante" a "muy importante"). El listado final incluía 30 indicadores, agrupados en "Indicadores Generales" (formación general, conocimiento del idioma, iniciativa-motivación...), "Indicadores para medir las habilidades en la búsqueda de empleo" (saber manejarse en un proceso de selección de personal o en un primer contacto con el empleador, conocer el mercado y sus oportunidades...), e "Indicadores relacionados con el empleo" (formación laboral, responsabilidad-compromiso...). Además, el cuestionario incluía algunas preguntas abiertas: por ejemplo, en el caso de los empleadores se incluían preguntas como: "A la hora de contratar inmigrantes, ¿qué es lo que más valora esta empresa?", o "En general, ¿cómo crees que vienen preparados los inmigrantes?". La pregunta abierta que se incluía en todos los cuestionarios era: “¿Qué necesita una persona inmigrante para conseguir un empleo en el País Vasco?".

A la hora de preparar la muestra se intentó abarcar todos los sectores relacionados con el empleo y la inmigración. Desde junio a diciembre del 2007 se pasó el cuestionario a inmigrantes, empleadores, sindicalistas, representantes de instituciones, asociaciones, expertos y formadores. Se recibieron 373 respuestas, de las cuales 212 (el 57\%) provenía de inmigrantes. De estos 212 el $45 \%$ provenía de Latinoamérica, el 31\% del norte de África (Marruecos, Argelia, Túnez...) y el 11\% de Europa del Este. Entre los no-inmigrantes, el $16 \%$ de los que han contestado han sido "Empleadores", el 11\% formadores, el 11\% sindicalistas... También se han realizado entrevistas en profundidad con informadores 


\section{Indicadores generales}

1. Formación general: saber leer, escribir, conocimientos matemáticos básicos, cultura general...

2. Conocimiento del idioma (castellano, euskera).

3. Iniciativa-motivación: tener un proyecto vocacional realis-

ta, perseverancia en lograr los objetivos, capacidad de iniciativa, motivación, saber tomar decisiones, capacidad de resolver problemas...

4. Personalidad-equilibrio emocional: control de los impulsos, resistencia a la frustración, estado de ánimo...

5. Salud en general: hábitos de limpieza, alimentación...

6. Relaciones familiares en general: clima afectivo en la familia, situación económica de la familia...

7. Habilidades sociales: normas básicas de educación, tener cortesía (saludo, agradecimiento...).

\section{Indicadores para la búsqueda de empleo}

8. Saber en qué se quiere trabajar de manera realista.

9. Grado de dedicación a la búsqueda de empleo y seguimiento de sus gestiones.

10. Tener prioridades.

11. Conocer el mercado y sus oportunidades.

12. Saber obtener información acerca de posibles empleos: conocer métodos-sistemas (acciones-estrategias) y lugares para la búsqueda de empleo.

13. Tener relaciones personales en el mercado.

14. Ser capaz de redactar un currículum.

15. Saber manejarse en un proceso de selección de personal o en un primer contacto con un empleador (saber autopresentarse, saber actuar en las entrevistas, tener suficiente autoestima....).

16. Tener actitudes favorecedoras (iniciativa, autonomía, persistencia, polivalencia, disposición a la actualización...).

\section{Indicadores relacionados con el empleo}

17. Formación laboral: conocimientos básicos de un oficio específico, habilidad para la utilización de herramientas, aparatos, tecnologías, tener experiencia laboral...

18. Ser capaz de planificar su trabajo: tener un ritmo adecuado de trabajo, saber gestionar el tiempo, cumplir los plazos previstos, saber concentrarse en el trabajo, cumplir horarios, ser constantes, disponibilidad...

19. Responsabilidad-compromiso: aceptar responsabilidades, actitud de compromiso.

20. Saber trabajar en equipo: tener una actitud de colaboración, saber compartir espacios y materiales, saber resolver conflictos con los compañeros...

21. Saber trabajar de forma autónoma.

22. Salvaguardar la salud laboral y respetar las normas en el trabajo.

23. Motivación-saber mantener la energía en el logro de un objetivo: identificar logros intermedios; saber motivarse; realizar esfuerzos para vencer los obstáculos y problemas que dificultan alcanzar un objetivo; desarrollar la capacidad para tolerar la frustración.

24. Buscar la calidad, el trabajo bien hecho.

25. Saber tomar decisiones, tener iniciativa, saber identificar y solucionar problemas.

26. Interés por aprender-formación continua: tener interés en su crecimiento profesional, identificar los conocimientos y las habilidades que se necesitan para actualizarse o mejorarse, destinar lugares y momentos para aprender y desarrollar nuevas habilidades.

27. Saber enfrentarse a nuevas situaciones flexiblemente: interpretar los hechos de forma flexible; manejar exigencia múltiples y prioridades cambiantes; adaptarse a los cambios.

28. Actuar creativamente: utilizar fuentes diversas para generar nuevas ideas; identificar oportunidades; aportar ideas innovadoras; encontrar formas nuevas y eficaces de hacer las cosas.

29. Saber traducir ideas en acciones: buscar apoyo y recursos para el desarrollo de una idea; desarrollar un plan de acción para concretar una idea.

30. Saber gestionar el desarrollo de la propia carrera: identificar las principales áreas de interés personal; definir objetivos y metas personales; determinar un plan de desarrollo de carrera; evaluar los avances del desarrollo de carrera. que el equipo investigador consideraba "especialmente importantes".

Entre enero y marzo del 2008 hemos analizado los cuestionarios (la parte cuantitativa del estudio a través del programa informático de estadística SPSS) y las entrevistas, y tras el análisis se ha vuelto a hacer una reunión con los expertos que trabajan con este tema para contrastar los resultados. En la primavera y verano del 2008 se han realizado las últimas reuniones entre los diferentes equipos de investigación (San Sebastián, Burdeos, Ámsterdam y Aviñón) para discutir las conclusiones conjuntas y las recomendaciones finales. 
TABLA 2. LISTA DE LOS INDICADORES MÁS IMPORTANTES PARA ENCONTRAR Y MANTENER UN EMPLEO

Indicador

Puntuación media

(1-nada importante, 6-importancia máxima)

1.- Tener los papeles en regla

5,04

2.-Tener ganas de trabajar, motivación

4,96

3.-Ser buena persona

4,85

4.-Saber presentarse entrevista

4,74

5.-Ser constante, insistir

4,67

6.-Tener buenas cualidades personales

4,63

7.-Saber castellano

4,60

8.-Saber conseguir información para buscar empleo

4,47

9.- Relacionarte con gente que te pueda ayudar

4,40

10.-Tener buena formación académica, escolarización

4,21

11.-Tener buena formación profesional

4,19

12.-Saber redactar el currículum

4,18

13.-Tener experiencia en el trabajo

4,18

14.-Saber cuál es el tipo de trabajo deseado

4,13

15.-Saber lograr información para crear una empresa

3,81

16.-Saber manejar internet

3,55

17.-Tener carné de conducir

3,48

18.-Saber vasco

3,15

En cuanto a las dificultades que nos hemos encontrado durante nuestra investigación, habría que destacar las siguientes: encontrar las personas adecuadas para el estudio, tanto en número como en los colectivos que necesitamos estén representados; afinar más en la tabla con los indicadores; conseguir una comprensión adecuada, principalmente en el caso de los inmigrantes, de los conceptos vertidos en la tabla de indicadores; obtener en las entrevistas una información rica y detallada, que en algunos casos se presenta de modo escueto y extremadamente simple y categorizar la información vertida en las entrevistas, tanto grabadas como escritas, para poder extraer una información representativa.

\section{Resultados}

Una vez analizadas las respuestas a los cuestionarios, ésta es la lista que resume la importancia de los indicadores según los encuestados (Tabla 2).
Un análisis más detallado de las respuestas cuantitativas y cualitativas nos permite clasificar las mismas con arreglo a las siguientes categorías:

\subsection{Cualidades representadas con amplio nivel de importancia}

Por las respuestas que se han obtenido con alto índice podemos afirmar que lo principal es "tener los papeles en regla y estar legalmente en el país de acogida":

En primer lugar, una de los grandes impedimentos que tiene un inmigrante es no tener la tarjeta de residencia por lo cual para acceder a un empleo es muy difícil (cuestionario num. 171, hombre, 30 años, procedencia: Chile).

Es decir, antes de pensar cualquier situación de formación profesional e inserción laboral, lógicamente lo que se necesita es un mínimo de estabilidad legal, cosa que es especialmente difícil para los inmigrantes. Ya hemos hecho referencia en la introducción 
al discurso crítico sobre la legislación española y europea. Pero viendo ahora los resultados, habría que volver a resaltar esta idea. En las reuniones con los expertos por ejemplo se ha hablado de la "picaresca de los contratos", porque hay inmigrantes que consiguen "contratos ficticios" pagándose a sí mismos, para poder conseguir de esta forma "papeles". El discurso crítico viene insistiendo en los últimos años en la discriminación que supone para los inmigrantes la legislación española ("la igualdad de oportunidades sólo es un principio recogido en la Constitución, en la realidad los inmigrantes lo tienen especialmente difícil por el mero hecho de ser inmigrantes"), a lo que ahora habría que añadir los primeros intentos de la UE para definir una política común en esta materia, política común que parece situarse también en la línea restrictiva y discriminatoria.

Respecto a la característica "ganas de trabajar y motivación" que hay que tener para encontrar un empleo, que aparece en segundo lugar de importancia en el total de las respuestas, podemos decir que para los inmigrantes este tema ocupa el tercer lugar, 4,94 puntos de media, mientras que para los autóctonos es el primero de los indicadores, con 4,97 puntos. Dentro del colectivo inmigrante, las mujeres puntúan este aspecto en segundo lugar, con 5,03 puntos y los hombres en cuarto nivel, con 4,88. Finalmente, los latinos dan más valor que el resto de los inmigrantes a las ganas de trabajar.

La motivación, las aspiraciones. Tener ganas de trabajar para mejorar (cuestionario num. 35, Formador CIP).

Estar dispuesto a trabajar en empleos que los vascos y españoles no quieren (cuestionario num. 28, mujer, 24 años, procedencia: Brasil).

Los aspectos relacionados con las cualidades personales, con la manera de ser o la personalidad ocupan muchas de las opciones en las respuestas de los inmigrantes. Por lo tanto, hay "una manera de ser", representada por un alto porcentaje de respuestas entre 4,60 y 5,00 puntos de media que define al inmigrante con cualidades para conseguir un empleo. Debe ser "buena persona", tener buena presencia y modales, simpatía, responsabilidad y no desanimarse:

Buena presencia (cuestionario num. 202, mujer, 23 años, procedencia: Argelia).

Tener simpatía, buena presencia y responsabilidad (cuestionario num. 28, mujer, 24 años, procedencia: Brasil).

Ser responsable y serio (cuestionario num. 193, hombre, 45 años, procedencia: Colombia).

Sobre todo para los propios inmigrantes, "ser buena persona" aparece por lo tanto como un indicador-competencia muy importante para conseguir y mantener un empleo. Hay que tener en cuenta que según los datos del Observatorio Vasco de Inmigración, la inmigración extranjera despierta en la sociedad vasca sentimientos encontrados y opiniones contradictorias. Por un lado, se reconoce que su llegada genera enriquecimiento cultural y económico. Otra cosa, es llevar ese reconocimiento teórico a la realidad cotidiana. De cerca, la opinión de los vascos revela, que existe un "preocupante" índice de opiniones y sentimientos de rechazo hacia los extranjeros. Por ejemplo, a la hora de valorar "el grado de simpatía hacia los inmigrantes, según su procedencia”, en una escala del o al 10 (siendo o-ninguna simpatía y 10-mucha simpatía), los vascos han dado una puntuación media de 3,92 al colectivo de "marroquíes". Tampoco llegan al cinco los "inmigrantes del África Negra", situándose en la parte alta de la escala los inmigrantes de la Unión Europea, con una puntuación media de $6,42^{5}$ (Observatorio Vasco de Inmigración, 2006). No es extraño por lo tanto que en las reuniones con la gente que trabaja en este campo se haya reforzado esta idea con el ejemplo de que los inmigrantes solicitan a veces a los técnicos del Servicio 
Público de Empleo-INEM que llamen a las empresas para explicar que el candidato es una "buena persona". Lo que vienen a demostrar esta clase de ejemplos es que a pesar de los discursos políticamente correctos, la sociedad vasca (como el resto de sociedades europeas) presenta todavía un alto grado de "desconfianza y rechazo" hacia los inmigrantes, y que lógicamente los propios inmigrantes son conscientes de esa desconfianza, por lo que necesitan demostrar (por encima y antes de su formación y experiencia profesional previa), que son "buenas personas".

En relación con lo anterior, "saber presentarse" es la siguiente característica más valorada por los encuestados. También en este caso los expertos han corroborado la importancia de este indicador-competencia, porque en los puestos de trabajo que vienen ocupando los inmigrantes, esta primera presentación suele ser más importante que el currículum. Así, a veces los inmigrantes han sido contratados tras presentarse directamente ante el empleador. Por eso, la relación con la gente, lo que los Técnicos Municipales llaman "crear la red" es muy importante en este y en otros temas. El "boca a boca" funciona todavía mejor que cualquier otro sistema para buscar trabajo, casa... Es necesario consultar periódicos, internet, etc., pero los técnicos que están trabajando en este tema aconsejan también el contacto directo, el hablar con otros inmigrantes, el ir directamente a las empresas, insistir y mostrar interés...

En relación con lo que estamos explicando, "ser constante, insistir en la búsqueda da empleo" y "tener buenas cualidades personales, simpatía, amabilidad, buena presencia, etc." son también indicadores o competencias que han sido valorados como muy importantes por los inmigrantes.

"Saber castellano" es una competencia que deben dominar quienes quieren conseguir un empleo, pero las valoraciones sobre este aspecto difieren mucho según quién es la persona que responde. Para los inmigrantes en su conjunto es un conocimiento que ocupa el sexto lugar, con 4,71 puntos, mientras que para los autóctonos se sitúa en octavo puesto $(4,47)$ dentro del colectivo de inmigrantes, las puntuaciones de los hombres y mujeres no difieren apenas, pero el origen de la persona inmigrante tiene una significación importante en esta respuesta. Como era de esperar, los latinos conceden menor importancia al dominio de la lengua castellana, al tiempo que los demás inmigrantes le dan una puntuación muy alta, de 5 puntos, colocándola en segundo lugar.

Hablar castellano correctamente, tanto para expresarse como para comprender órdenes (128, INEM-SPEE).

\subsection{Cualidades representadas con un porcentaje medio de importancia}

"Saber conseguir información". Indicador o competencia que ha sido valorada sin grandes variaciones entre los distintos grupos.

Importancia del papel facilitador de la red informal en la búsqueda de empleo (familias contactos, amigos...) (cuestionario num. 41, Técnico del Ayuntamiento de Azkoitia).

Contactos con gente que trabaje en algún organismo (cuestionario num. 202, mujer, 48 años, procedencia: Colombia).

"Tener relaciones con gente que te puede ayudar a conseguir un empleo" puntúa de manera diferente según los colectivos y grupos analizados. Para los inmigrantes parece ser más importante que para los autóctonos. Por otra parte, dentro del colectivo inmigrante son los hombres quienes más valoran esta cualidad. Entre los inmigrantes, los latinos son quienes le dan la puntuación más alta a esta competencia analizada.

"Formación y experiencia". Una buena formación profesional es altamente valorada por los inmigrantes, mientras que los autóctonos le dan menos importancia. Las mu- 
jeres y los hombres valoran de modo similar y los no latinos puntúan por encima de los latinos.

"Redactar el currículo y saber lo que se quiere". Saber redactar el currículo está valorado con 4,18 por la muestra total analizada, pero sube hasta el 4,40 en el caso de los inmigrantes y solamente alcanza un 3,90 cuando quien responde es una persona no inmigrante. Dentro del colectivo inmigrante, las respuestas no difieren en gran medida entre hombres y mujeres y entre latinos y no latinos.

"Tener experiencia en el trabajo" es valorado en mayor medida por los inmigrantes sobre los autónomos. Hombres y mujeres puntúan casi igual. Los no latinos valoran esta característica por encima de los latinos.

"Saber el tipo de trabajo que se quiere conseguir" tiene una mayor valoración entre los inmigrantes que entre los autóctonos encuestados. Dentro del colectivo inmigrante, apenas hay diferencias significativas entre los grupos de hombres y mujeres, o entre latinos y no latinos.

Por otra parte, el "saber conseguir información para crear su propia empresa” ocupa el último lugar de este bloque intermedio $(3,81)$. Saber conseguir información para crear su propia empresa tiene mucha menos importancia para los autóctonos que para los inmigrantes. Hombres y mujeres, así como latinos y no latinos valoran de modo similar este aspecto.

\subsection{Cualidades representadas con un bajo por- centaje de importancia}

En este último bloque encontramos las cualidades que menor valoración han recibido. "Tener el permiso de conducir" está mucho más valorado entre los inmigrantes $(3,93)$ que entre los autóctonos $(2,89)$. Dentro del colectivo inmigrante, son las mujeres quienes le dan mayor importancia que los hombres, y los no latinos que los latinos.
De igual modo, el "manejo de las nuevas tecnologías" con una media de 3,55 refleja una relativamente menor importancia por parte de los encuestados. "Saber manejar Internet" es una competencia que valoran mucho más los inmigrantes $(3,92)$ que los autóctonos $(3,06)$. Dentro del colectivo inmigrante, los hombres aprecian más esta cualidad que las mujeres, mientras que los latinos y no latinos puntúan de modo similar.

"Saber vasco o euskera" es una competencia que valoran mucho más las personas inmigrantes $(3,44)$ que las autóctonas $(2,79)$. Dentro del colectivo inmigrante, los hombres aprecian más esta característica que las mujeres y finalmente, son los latinos quienes le asignan una puntuación más alta que los no latinos. Ocupa el último lugar en la lista de prioridades a la hora de valorar la importancia de las cualidades y competencias de las personas inmigrantes a la hora de conseguir un empleo.

Una de las conclusiones del estudio cuantitativo es que todos los indicadores o competencias han sido valorados como más importantes por los inmigrantes que por los autóctonos. Lo que plantea una posible hipótesis a analizar en futuras investigaciones: "los nacidos en el País Vasco creen que no es tan importante que los inmigrantes tengan un buen currículum, que tengan experiencia previa, que conozcan idiomas, etc., porque consciente o inconscientemente los queremos dirigir a los peores empleos, a los que nosotros no queremos, a los que quedan libres. Por eso, no nos importa que no tengan tanta formación. Incluso puede que esto sea bueno (para "nosotros"), porque a nadie le gusta que "le muevan la silla", y si a eso añadimos el miedo o la desconfianza al diferente...."

\subsection{Otras respuestas abiertas}

"Condiciones de acogida". Destacamos que una de las condiciones de acogida iniciales 
es poder contar con la ayuda y asesoramiento para integrarse y obtener ayudas básicas.

Además, según destacan diversos responsables sindicales y agentes sociales, es necesaria cierta dosis de "solidaridad". Hace falta también que las empresas aprendan a confiar en los inmigrantes. En muchos casos, lo que piden los inmigrantes es simplemente una oportunidad para poder demostrar que saben y quieren trabajar. También se destaca como importante el poder disponer de unas condiciones de habitabilidad óptimas.

"Sensibilizar a la sociedad". Por las respuestas obtenidas, los encuestados valoran que la sociedad en su conjunto debe tomar conciencia de la nueva realidad y sensibilizarse para dar nuevas respuestas que permitan una mejor integración de los inmigrantes. Por lo tanto, empresas, formadores, agentes sociales y ciudadanos en general debieran adaptarse a los nuevos tiempos.

Además de los aspectos laborales y profesionales, existen otras facetas de la vida de los inmigrantes que es necesario tener en cuenta:

"Respetar sus culturas. No meterse con su personalidad" (cuestionario num. 43, hombre, 25 años, procedencia: Argelia).

\section{Conclusiones}

Teniendo en cuenta los resultados de nuestro estudio, podríamos decir que existen dos grandes bloques de conclusiones: en primer lugar, las medidas o iniciativas que deben ser tenidas en cuenta como requisitos previos o condiciones básicas que deben existir para que los inmigrantes puedan acceder a un empleo y en segundo lugar, aquellos aspectos que pueden ser directamente desarrollados en cursos de formación para personas inmigrantes.

\subsection{Condiciones básicas y requisitos previos}

"Reformas legales". En contra de lo que habitualmente parece ocurrir, no es solamente el colectivo inmigrante quien tiene que adaptarse a las demandas que la sociedad de acogida tiene respecto a las materias relacionadas con el empleo. También la sociedad vasca debe cambiar en algunos aspectos y facilitar la acogida y la integración de los inmigrantes. En esta investigación constatamos que, tal y como previamente habíamos mostrado en la primera parte del estudio, existen una serie de impedimentos legales que deben ser superados para poder establecer con cierta normalidad un campo de actuación en el terreno de la consecución del empleo.

"Mejorar las condiciones de acogida". Estas condiciones básicas hacen referencia a diversos campos de actuación: contar con algún tipo de ayuda y asesoramiento para que los inmigrantes tengan una más fácil llegada al país de acogida; es necesaria también cierta dosis de solidaridad y confianza con los inmigrantes; en algunos casos, lo que piden los inmigrantes es simplemente una oportunidad; finalmente, una condición indispensable es una vivienda.

"Sensibilizar a la sociedad". Por las respuestas recibidas, muchas de las personas encuestadas subrayan que es la sociedad en su conjunto quien debe tomar conciencia de la nueva realidad y sensibilizarse para dar nuevas respuestas que permitan una mejor integración de los inmigrantes. Por lo tanto, hay que insistir en la necesidad de que empresas, formadores, agentes sociales y sociedad en general deben adaptarse a los nuevos tiempos.

"Respetar la identidad de los inmigrantes". Finalmente, los inmigrantes reclaman ser considerados como personas con una cultura y lengua propia y que sean tenidas en cuenta y no se les considere únicamente como mano de obra dispuesta incondicionalmente al trabajo. 


\subsection{Aspectos relacionados con la formación}

"Aspectos relacionados con la motivación". En este terreno, son aspectos fundamentales los siguientes: tener en cuenta las posibles actividades que pueda desarrollar y a tener una visión clara de cuáles son las oportunidades más frecuentes; definir con la mayor claridad posible el tipo de trabajo que se quiere hacer; orientar hacia empleos que pueden encajar mejor con sus cualidades y su formación.

"Cualidades de tipo personal y cultural". Desarrollar habilidades sociales, cuidar aspectos personales como la responsabilidad, autoestima, resistencia al desánimo, etc. debieran formar parte de la formación que deben recibir quienes buscan un empleo en nuestra sociedad. Por ejemplo, cuando hablamos de responsabilidad, nos referimos a que los inmigrantes deben conocer la cultura general y laboral de nuestra sociedad (ritmo de trabajo, puntualidad...). Pero en este punto hay que tener en cuenta que también hay competencias culturales que se deben trabajar también entre los que han nacido en el País Vasco: la lucha contra las diferencias de género, el conocimiento y respeto de la diversidad cultural, etc. (por ejemplo, si los latinoamericanos en general son "más suaves en las formas y más diplomáticos" que los vascos, también éstos deberían hacer un esfuerzo para no resultar "demasiado directos o bruscos" en sus relaciones profesionales con este colectivo).

"Competencias instrumentales". Competencia en lengua castellana y vasca, competencias de búsqueda y relación, competencia en redactar el currículo y saber presentarse...

"Competencias profesionales" (formación y experiencia). Los cursos de formación deben cuidar, obviamente, aquellos aspectos más relacionados con la adquisición de conocimientos y preparación para los empleos a los que se va a dirigir la persona inmigrante. Por lo tanto, estos cursos deberán de- sarrollar entre estas personas una buena formación general, una adecuada cualificación profesional específica para los empleos y además deberán facilitar las prácticas o experiencias que permitan adquirir destrezas en el trabajo específico que se trate.

"Competencias complementarias". Dentro del capítulo de las competencias de tipo profesional, existen dos aspectos que, aunque en menor grado de importancia, pueden tener eco en los programas de formación: la obtención del tener carné de conducir, que puede facilitar la consecución de determinados empleos, y el conocimiento y manejo de las nuevas tecnologías, que son necesarias cada vez en mayor medida en determinados trabajos. En este sentido, un ejemplo puede ser la necesidad de aprender a manejarse en una sociedad burocrática como la nuestra: si se necesita ir al médico y por lo tanto se van a perder algunas horas de trabajo hay que pedir permiso; si se tienen problemas laborales se puede pedir ayuda en los sindicatos... Son cuestiones evidentes para los que hemos nacido en nuestras sociedades industriales-capitalistas, pero no tanto para inmigrantes provenientes de otras sociedades y culturas. En este sentido, los expertos del ámbito de los servicios sociales han comentado en este estudio el interés de una posible figura de "acompañamiento-asesor-tutor", sobre todo (lógicamente) en los primeros momentos de la llegada al país de acogida. 


\section{Referencias bibliográficas}

Adam Muñoz M. D. y Jiménez Sánchez G. (2006): La educación y la formación profesional de los inmigrantes. Córdoba: Servicio de Publicaciones de la Universidad de Córdoba.

Álvarez I. (2000): Integración en la sociedad: ¿quién tiene que cambiar más? Donostia-San Sebastián: SOS Racismo de Guipúzcoa.

Comisión de Ayuda al Refugiado en Euskadi (2006): Inmigración y Empresa. Vitoria: Gobierno Vasco.

Izquierdo A. (2003): "Mercado laboral e inmigración”, en Garrido V. (coord.): Sociedad civil e inmigración. Barcelona: Ariel.

Ministerio de Trabajo y Asuntos Sociales. Secretaría de Estado de Inmigración y Emigración. Dirección General de Integración de los Inmigrantes (2007): Plan Estratégico de Ciudadanía e Integración 2007-2010 (http://extranjeros.mtas.es)

Observatorio Vasco de Inmigración (2006): Percepciones, valores y actitudes de la población vasca hacia la inmigración extranjera (www.ikuspegi.org)

Solé C. (2001): El impacto de la inmigración en la economía y en la sociedad receptora. Barcelona: Anthropos.

Solé C. (2006): Inmigración comunitaria: ¿discriminación inversa? Barcelona: Anthropos.

\section{Notas}

${ }^{1}$ Investigación financiada en el marco del programa comunitario SÓCRATES, acción GRUNTVIG 2 (convenio financiero o7-ESPo1-GRo4-00155-2, 20062008). Han participado la Universidad del País Vasco, la Hogeschool van Amsterdam, la asociación AIFRISS de Burdeos ("Association Internationale de Formation et de Recherche en Instruction Spécialisée, Santé et Social") y la Asociación CRIDF PACA, de Aviñón, Francia. En este texto presentamos la investigación realizada en el País Vasco.

${ }^{2}$ San Sebastián cuenta desde el año 2005 con su propio Plan Municipal de Inmigración, "Donostia Elkarrekin" (“Juntos San Sebastián”)

${ }^{3}$ www.ikuspegi.org
${ }^{4}$ Para una visión crítica de la legislación española y europea en materia de inmigración nos parece especialmente interesante en el caso guipuzcoano la labor de la ONG "Sos Racismo" (Sos Arrazakeria en vasco): www.sosracismo.org

${ }^{5}$ Con respecto a la relación entre diferentes colectivos de inmigrantes y el mercado laboral, de las reuniones con expertos podríamos extraer el siguiente esquema: cuando los magrebíes vienen con algún tipo de formación previa, ésta suele generalmente ser lo que en España denominaríamos "Formación Profesional" (FP), por lo que podrían encontrar empleos en sectores como el de la construcción. Pero como ya hemos indicado, en este colectivo tiene mucha importancia el estereotipo previo, la imagen (negativa) socialmente construida, muchas veces relacionada con la diferencia cultural y especialmente la diferencia religiosa (cristianismo-islam). Los expertos han definido al colectivo de inmigrantes latinoamericanos como un colectivo "menos formado, poco entregado al trabajo (según los empleadores) pero con mayor cercanía cultural". Por último, otro numeroso grupo de inmigrantes sería el de los "europeos del este", "muy formados (lo que a veces crea sospechas: ' $¿ \mathrm{es}$ posible que todos sean médicos o ingenieros?") y los más cercanos a nuestra cultura laboral".

DiRECCIÓN DE LOS AUTORES: Félix Etxeberria. Universidad del País Vasco. Facultad de Filosofía y Ciencias de la Educación, Departamento de Teoría e Historia de la Educación. Avenida de Tolosa, 70. 20018 Donostia.

Correo electrónico: fetxeberria@ehu.es

Fecha de recepción del artículo: 01.II.2008

Fecha de aceptación definitiva: o6.v.2008 